\title{
Diagnosing Emerging Infectious Diseases of Trees Using Ground Penetrating Radar
}

\author{
Iraklis Giannakis, Fabio Tosti, Senior Member, Livia Lantini, Amir M. Alani
}

\begin{abstract}
Ash dieback, acute oak decline (AOD) and Xylella Fastidiosa are Emerging Infectious Diseases (EIDs) that have spread rapidly in European forests during the last decade. Quarantine measurements have mostly failed to repress the outbreaks and millions of trees have already been infected. Identifying infected trees in a non-destructive manner is of high importance for monitoring, managing and preventing EIDs. The aim of this paper is to examine the capabilities of Ground Penetrating Radar (GPR) on evaluating the internal structure of tree-trunks and detecting tree-decay associated with EIDs. Traditionally used processing schemes tuned for GPR line-acquisitions are modified accordingly to be compatible with the new measurement configurations. In particular, a detection framework is presented based on a modified Kirchhoff and a reverse-time migration. Both of the aforementioned methodologies are compatible with measurements taken along closed irregular curves assuming a homogeneous permittivity distribution. To that extent, prior to migration, a novel focal criterion is used that estimates the bulk permittivity of the host medium from the measured B-Scans. The suggested detection scheme is successfully tested on both numerical and laboratory measurements, indicating that GPR has the potential to become a coherent and practical tool for detecting tree-decay associated with EIDs.
\end{abstract}

Index Terms-Ash dieback, acute oak decline, AOD, emerging infectious diseases, EIDs, forestry, ground penetrating radar, GPR, Kirchhoff, migration, reverse-time migration, signal processing, tree, trunk, Xylella Fastidiosa.

\section{INTRODUCTION}

W OODLANDS and forests have undergone significant changes the last century due to invasive Emerging Infectious Diseases (EIDs) caused by pathogens, pests and fungi [1], [2]. Recent examples include the European beech decline [3], the chestnut blight that almost brought the chestnut trees of North America to an extinction [4], [5] and the dutch elm disease that caused significant damage to the elm forests in central Europe [6]. Clear evidences suggest that modern socioeconomic factors such as accelerating human population [7], international travelling [2], global timber trade [1] and artificial erosion of geographic barriers [2], have largely increased the spreading rate of invasive pathogens [7]. In addition, climate change has raised the global temperature which has further contributed to the spreading of EIDs [2], [3].

The reasons above led to an exponential increase of EIDs [1]. It is indicative that from 1995-2010, EIDs have showed a 13-fold increase [8]. The most prominent of them are the ash dieback, the acute oak decline (AOD) and the Xylella

Iraklis Giannakis, Livia Lantini, Fabio Tosti and Amir Alani are with the school of computing and engineering, University of West London, London, W5 5RF, Iraklis.Giannakis@uwl.ac.uk, Fabio.Tosti@uwl.ac.uk, Livia.Lantini@uwl.ac.uk, Amir.Alani@uwl.ac.uk.
Fastidiosa. Ash dieback is a major threat to European ash forests [9] with high susceptibility [10] and mortality rates [9]. It was confirmed in UK in 2012 [1] and it has rapidly spread since then mostly in Wales and southeast England [9]. Apart from ash dieback, AOD is another recently introduced EID that poses a major threat to European oak populations [11]. AOD is a multi-agent EID that has spread rapidly in the UK the last decade [11]. The progression of the disease is particularly fast and can lead to tree-mortality within 3-5 years [11]. Ash dieback and AOD have already infected thousands of trees in the UK, nonetheless their impact is dwarfed in comparison to the millions of trees affected by Xylella Fastidiosa in Italy [13]. Xylella Fastidiosa is a vector-transmitted, slow progressing bacterium [14], [15] that has spread in Italy with devastating effects to the overall population of olive trees [13]. Italy has declared state of emergency since 2015 and is now under European quarantine control [16]. The international scientific community and the media [17] have underlined that under the current framework, eradication of EIDs is a laborious task and new forestry approaches should be developed for monitoring and diagnosing EIDs [2].

Tree decay and compartmentalization of decay (CONDIT) are non-visible structures associated with the overall health and structural integrity of trees [18], [19], [20], [21], [22], [23]. Drilling approaches for measuring the compactness [24] and the electrical conductivity [25] of the inner trunk have been widely employed for detecting decay. Destructive methods, although reliable, they are time-consuming and they can cause irreversible damage to the outer bark making the tree more susceptible to pathogens, fungi and pests. Consequently, nonintrusive methodologies are off high importance when it comes to monitoring and containing EDIs and recent developments on remote sensing have shown promising results on that front [17].

Exploration geophysics and non-destructive testing (NDT) for wood monitoring [26], [27] can provide efficient and accurate diagnostic tools against EIDs. The most mainstream NDT methods applied for wood monitoring are the electrical resistivity tomography [28], [29], [30], [31], the acoustic tomography [32], [33] and ground penetrating radar (GPR) [34], [35], [36], [37]. Regarding GPR, microwave imaging using wide angle refraction measurements [40] and raybased tomography [41] have shown promising results under laboratory conditions. Microwave imaging requires multiple measurements using bespoke antenna systems with separate transmitters and receivers. This configuration differs from typical GPR surveys, which they usually rely on common-offset (CO) commercial antennas [42]. The latter are particularly appealing due to their minimum computational and operational 
requirements making them suitable for large scale forestry applications [43].

In the context of CO-GPR, -and in an effort to develop commercially appealing and practical methodologies- a polarbased interpretation of B-Scans is presented in [34], [37], [38], a layer-based classification is described in [39] and a signal processing scheme for estimating the origins and the sizes of the decay is proposed in [43]. The main drawback of the aforementioned techniques is that they require manual processing and characterization. Due to that, the interpretation is relied on the experience and the expertise of the GPR practitioner on correctly identifying and characterizing reflection patterns associated with potential decay. To tackle this, a semi-automatic migration-based framework using CO-GPR configuration is proposed in this paper.

Migration has been initially applied in seismic surveys [44] and has been successfully extrapolated to GPR [42] due to the similarities between GPR and seismic wave phenomena. From landmine detection [45], [46], [47] and cross-borehole surveys [48] to surface profiling [49] and target detection in multilayered scenarios [50], migration has proven its status as one of the most popular interpretation tools in the GPR community primarily due to its accuracy and minimum computational requirements. Regarding measurements along closed curves, Kirchhoff migration has been applied for column investigation [51] and preliminary results are given on the applications of linear inversion subject to a cylindrical host medium [52], [53]. Kirchhoff migration is an appealing choice for tree investigations due to its flexibility on dealing with varying topography and irregular measurements [54], [55]. In the current paper, a scaled Kirchhoff migration is used in order to successfully reconstruct the scattering sources within the trunk assuming a homogeneous velocity. To complement Kirchhoff migration, a reverse-time (RT) migration is used in an effort to detect early decay in scenarios with low signal to clutter ratio. The employed RT-migration uses a second order in both space and time finite-difference time-domain (FDTD) method [59], [60] to back-propagate the measured fields. RT migration is a powerful reconstruction technique that has gained its reputation through its flexibility on dealing with complex media subject to irregular measurements [61], [62]. RT migration can be applied to both wide-angle [48] and COGPR configurations [63] and when it is coupled with FDTD can deal with anisotropic materials with arbitrary distributions in a straightforward manner [61]. For the current problem, the velocity structure of the trunk is not trivially available. Consequently, the velocity of the trunk is assumed to be homogeneous and it is estimated using auto-focusing criteria [56], [57], [58].

Migration is a linear imaging method that assumes point scatterers acting as impressed sources initiated at time zero [44]. Repetitive reflections (ringing noise) between tree-layers and cross-coupling violate these conditions and introduce artifacts and clutter to the reconstructed image. Singular value decomposition filter (SVD) [64] has proven very effective on dealing with ringing noise on tree surveys [43] and it is applied here as part of a pre-processing step prior to the migration. In addition, the post-migrated data are squared and smoothed in order to increase the overall signal to clutter ratio.

The aim of this paper is to develop a semi-automatic processing scheme that can accurately detect the presence of early decay in trees. To achieve this aim, a multi-stage data processing methodology is proposed based on the aforementioned sequential steps i.e. pre-processing, migration and postprocessing, combined with the wheel-based positioning described in [43]. Both numerical and laboratory measurements are used to support the premise that CO-GPR commercial antennas coupled with the proposed detection scheme can become a robust diagnostic tool against EIDs.

\section{Methodology}

The main core of the proposed methodology is the qualitative reconstruction of the internal structure of trees using Kirchhoff [54] and RT migration [61]. These methods complement each other and they provide robust and efficient interpretation tools for tree-surveys. The measurement configuration applied throughout the paper consists of parallel scans orthogonal to the main axis of the trunk. Prior to migration, every A-Scan should be assigned with a specific set of coordinates $(x, y) \in \mathbb{R}$. Commercial CO-GPR systems utilize a wheel-measuring device to position each A-Scan. This approach is suitable for line measurements in halfspaces but is not applicable when measurements are taken on irregular surfaces [43]. To that extend -and similar to [43]- an arc-length parametarization [66], [67] is used here in order to transform the wheel-based measured distance to 2D coordinates.

Initially, the shape of the trunk is digitized $\{x, y \in$ $\mathbb{R} \mid x, y>0\}$ using $n$ points along the surface of the trunk. Spline interpolation is then employed in order to map the coordinates to the parametric variable $\left\{t \in \mathbb{R}^{n} \mid t \in[0,1]\right\}$. This results to a continuous vector $\mathbf{F}=\langle P(t), L(t)\rangle$ that relates the coordinates of the investigated complex-shape to the arbitrary parametric variable $t$. We want to express the vector $\mathbf{F}$ with respect to the distance from a given reference point such as to be consistent with the typical measurement configuration applied in CO-GPR acquisitions. To that extend, we evaluate the arc-length of the vector $\mathbf{F}$ with respect to $\tau \in[0,1][43]$

$$
s(\tau)=\int_{0}^{\tau}\left\|\frac{d \mathbf{F}}{d t}\right\| d t=\int_{0}^{\tau} \sqrt{\left(\frac{d P(t)}{d t}\right)^{2}+\left(\frac{d L(t)}{d t}\right)^{2}} d t
$$

The integral in (1) is evaluated numerically [43] for different values of $\tau$ and subsequently a spline interpolation is used to map the values of $\tau$ with respect to $s$. Therefore, from a given distance $s$, its equivalent $t$ can be calculated. Thus, the position of every point on the investigated surface can be written in a vector format as $\mathbf{F}=\langle P(t(s)), L(t(s))\rangle$, where $P(t(s))$ and $L(t(s))$ are real scalar functions that represent the coordinates on the surface of the trunk with respect to the distance $\{s \in \mathbb{R} \mid s>0\}$ from a reference point.

Subsequently, a pre-processing step is executed that includes a zero-offset removal, a time-zero correction, a linear timevarying gain and an SVD filter [64]. For the case-studies examined in this paper it has been derived that filtering out two 
to twelve dominant eigenvalues sufficiently removes ringing noise and cross-coupling phenomena. For more details regarding the pre-processing step and the arc-length parametarization the reader is referred to [43].

The filtered B-Scans are now in a suitable format for the modified Kirchhoff and the RT migration. Although special care is taken in the pre-processing step to remove unwanted signal, nonetheless, the reconstructed internal structure of the trunk is still corrupted with migration-artifacts and remaining clutter. In an effort to further increase the overall signal to clutter ratio and facilitate interpretation, the post-migrated images are squared and subsequently smoothed using a Gaussian blur filter [65]. The above processing pipeline consists of sequential steps with minimum computational requirements. Pre-processing, migration and post-processing can be executed in mainstream computers within reasonable time which makes the suggested framework commercially appealing for large scale forestry applications.

\section{A. Modified Kirchhoff Migration}

The Kirchhoff integral for $t=0$ and subject to an arbitrary closed surface is given by [54]

$$
Q\left(\mathbf{r}_{m}\right)=\frac{1}{2 \pi} \oiint_{S_{0}} \frac{\partial R}{\partial n}\left(\frac{1}{R^{2}} W\left(\mathbf{r}, \frac{R}{v}\right)-\frac{W^{\prime}\left(\mathbf{r}, \frac{R}{v}\right)}{v R}\right) d S
$$

where $W(\mathbf{r}, t)$ is the filtered B-Scan measured at the position $\mathbf{r}=\langle x, y, z\rangle$ and $W^{\prime}(\mathbf{r}, t)=\frac{\partial W(\mathbf{r}, t)}{\partial t}$ is the derivative of the filtered B-Scan with respect to time $t$. The vector $\mathbf{r} \in S_{0}$ where $S_{0}$ is a closed surface in the 3D space. The reconstructed image is denoted as $Q\left(\mathbf{r}_{m}\right)$ where $\mathbf{r}_{m}=\left\langle x_{m}, y_{m}, z_{m}\right\rangle$ are the Cartesian coordinates of the investigated point. The distance between the investigated point $\mathbf{r}_{m}$ and a point at the surface $S_{0}$ is given by $R=\left\|\mathbf{r}-\mathbf{r}_{m}\right\|$. The velocity of the medium $v$ is assumed to be homogeneous and is given in $\mathrm{m} / \mathrm{s}$. Notice that the velocity $v$ used in (2) is half the actual velocity in order to compensate for the two-way travel time present in CO-GPR measurements. Lastly, the derivative $\frac{\partial R}{\partial n}$ is the derivative of the distance $R$ on the direction normal to the surface $S_{0}$.

In the current paper, the measurements are taken along the surface of the tree on equidistant and parallel scans with respect to the ground. Consequently, the measurement-grid is dense along the circumference and sparse along the main axis of the trunk. Due to that, 3D migration will have negligible advantages compared to pseudo-3D migration consisted of 2D migrated slices on the $x, y$ plane (parallel to the ground) [68]. In that context, and in order to reduce the complexity and keep the algorithm computationally efficient, a 2D migration is performed for each circular scan and the results are combined to a pseudo-3D image. The generalized Kirchhoff integral for 2D is now evaluated over the closed curve $T_{0}$, a continuous and differentiable function that lies on the $x, y$ plane. The vectors $\mathbf{r}_{m}$ and $\mathbf{r}$ are now fixed at the $z$ axis.

Utilizing the arc-parametarization of the trunk [43], $T_{0}$ is approximated using the vector $\mathbf{F}=\langle P(s), L(s)\rangle$. Notice that $\mathbf{r}$ denotes a 3D set of points that lie along the curve $\mathbf{F}$. Therefore, the Kirchhoff integral for a 2D curve $T_{0}$ along a constant depth
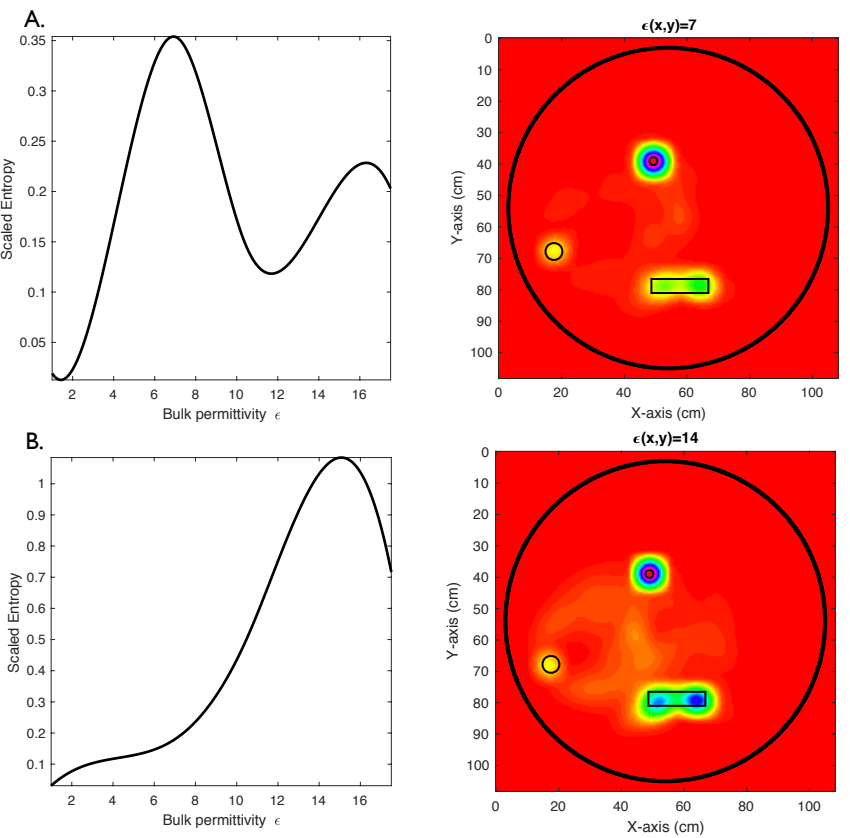

Fig. 1. Two case studies used to illustrate the capabilities of the scaled entropy on estimating the bulk permittivity of the host medium. Three low dielectric targets $(\epsilon=2)$ are buried in a homogeneous medium with A) $\epsilon=7$ and B) $\epsilon=14$. The targets are illustrated with solid black lines. The right images illustrate the migrated data using the velocities estimated based on the scaled entropy (9). The left images show the scaled entropy (9) calculated for different $\epsilon \in[1,18]$.

$z$ can be re-written as

$$
Q\left(\mathbf{r}_{m}\right)=\frac{1}{2 \pi} \int_{0}^{M} \frac{\partial R}{\partial n}\left(\frac{1}{R^{2}} W\left(\mathbf{F}, \frac{R}{v}\right)-\frac{W^{\prime}\left(\mathbf{F}, \frac{R}{v}\right)}{v R}\right) d s
$$

where now $R(s, m)=\left\|\mathbf{r}_{m}-\mathbf{F}\right\|$ and $M$ is the circumference of the tree i.e. the maximum distance $s$ from the reference point.

Via interpolation, the variable $W\left(\mathbf{F}, \frac{R}{v}\right)$ in (3) can be easily evaluated in a continuous manner since each A-Scan is properly positioned with respect to the distance $s$ using the arcparametarization approach described in [43]. The derivative $W^{\prime}\left(\mathbf{F}, \frac{R}{v}\right)$ is evaluated numerically using a second order finite-difference scheme. The directional derivative $\frac{\partial R}{\partial n}$ denotes the derivative of the distance $R$ on the direction normal to the curve $\mathbf{F}$. Therefore, $\frac{\partial R}{\partial n}$ can be written as $\frac{\partial R}{\partial n}=\nabla R \cdot \mathbf{n}$ [69], where $\mathbf{n}=\frac{\mathbf{A}}{\|\mathbf{A}\|}$ is the unit vector orthogonal to $\mathbf{F}$ i.e. $\mathbf{A}=\left\langle\frac{\partial L(s)}{\partial s}, \frac{-\partial P(s)}{\partial s}\right\rangle$ [69]. Lastly, the velocity $v$ is estimated using the focal criterion described in Section II.C.

The integral in (3) can be evaluated numerically in a straightforward manner with minimum computational requirements. The numerical evaluation of (3) can be seen as a diffraction summation scaled with respect to the distance $R$ and to the directional derivative $\frac{\partial R}{\partial n}$. The latter, increases the contribution from the segments in $\mathbf{F}$ that are aligned with the orthogonal lines to $\mathbf{F}$ that pass from the investigated point $\mathbf{r}_{m}$. Through this, directivity aspects are incorporated in the migration making the process more robust and accurate. Scaling the diffraction summation with respect to $R$ increases the contribution of early reflections and masks deeper structures. 
In order to overcome this a modified Kirchhoff migration is suggested

$Q\left(\mathbf{r}_{m}\right)=\frac{R_{m}}{2 \pi} \int_{0}^{M} \frac{\partial R}{\partial n}\left(\frac{1}{R^{2}} W\left(\mathbf{F}, \frac{R}{v}\right)-\frac{W^{\prime}}{v R}\left(\mathbf{F}, \frac{R}{v}\right)\right) d s$

where $R_{m}=\min _{s \in[0, M]} R(s, m)$. This implies that every diffraction summation is scaled with respect to a normalized distance. The distance is normalized subject to the minimum distance $R(s, m)$ between the investigated point $\mathbf{r}_{m}$ and the closed curve $\mathbf{F}$. Using this approach, each diffraction curve is independently scaled based on its minimum distance from $\mathbf{F}$. In the original Kirchhoff migration (3), late reflections are repressed in general regardless of the diffraction curve. In the modified scheme (4), late reflections with respect to the diffraction apex are repressed. Thus, scaling still occurs but it is no more biased to shallow targets.

\section{B. Reverse-Time Migration}

Maxwell's equations for linear, non-dispersive and nonmagnetic media are given by [70]

$$
\begin{gathered}
\nabla \times \mathbf{H}=\epsilon \frac{\partial \mathbf{E}}{\partial t}+\sigma \mathbf{E}+\mathbf{J} \\
\nabla \times \mathbf{E}=-\mu_{0} \frac{\partial \mathbf{H}}{\partial t} \\
\nabla \cdot(\epsilon \mathbf{E})=\rho \\
\nabla \cdot\left(\mu_{0} \mathbf{H}\right)=0
\end{gathered}
$$

where the vectors $\mathbf{E}=\left\langle E_{x}, E_{y}, E_{z}\right\rangle$ and $\mathbf{H}=\left\langle H_{x}, H_{y}, H_{z}\right\rangle$ are the electric and the magnetic fields respectively, $\sigma$ is the conductivity, $\epsilon$ is the permittivity, $\mu_{0}$ is the magnetic permeability of free space, $\rho$ is the charge intensity per cubic meter and $\mathbf{J}=\left\langle J_{x}, J_{y}, J_{z}\right\rangle$ represents the impressed current density [60].

In RT-migration for CO-GPR, the filtered B-Scan $W(\mathbf{F}, t)$ for the interval $\left\{\left[0, t_{\max }\right] \in \mathbb{R} \mid t_{\max }>0\right\}$ is initially reversed with respect to time $W\left(\mathbf{F}, t_{\max }-t\right)$. Subsequently, the reversed measurements are used as inputs for the impressed current sources $J_{u}(\mathbf{F}, t)=W\left(\mathbf{F}, t_{\max }-t\right)$, where $u \in\{x, y, z\}$ is the polarization of the receiver. Notice that the forward model must be evaluated only one time since the impressed sources $J_{u}$ are excited simultaneously and not in a sequential manner like a typical B-Scan. This greatly reduces the computational time necessary for the backpropagation of the received signals. Similar to Kirchhoff migration, the velocity used in the RT migration is half the actual velocity in order to effectively simulate the two-way travel-time. Using the revised velocity structure and the reversed current sources, the electric and the magnetic fields are back-propagated until the diffractions collapse to their origin at $\mathbf{E}\left(\mathbf{r}_{m}, t=0\right)$ [61].

In the proposed scheme, the fields are back-propagated using a second order TM-FDTD [59], [60]. A 2D configuration is chosen in order to further reduce the computational requirements. A TM-FDTD consists of $E_{z}, H_{x}, H_{y}$. Thus, the polarization of the antenna is assumed to be parallel to the main axis of the tree and perpendicular to the curve of measurements $T_{0}$.
TABLE I

The Extended Debye Properties of the Tree Layers [43]

\begin{tabular}{rccccc}
\hline Name & $W C$ & $\epsilon_{\infty}$ & $\Delta \epsilon$ & $\sigma\left(\Omega^{-1} m^{-1}\right)$ & $t_{0}(\mathrm{sec})$ \\
\hline Cabdium layer & $40 \%$ & 9 & 43 & 1 & $9.23 \mathrm{e}-12$ \\
Outer Sapwood & $30 \%$ & 6.1 & 12.36 & 0.033 & $9.23 \mathrm{e}-12$ \\
Inner Sapwood & $25 \%$ & 5.9 & 9.66 & 0.02 & $9.23 \mathrm{e}-12$ \\
Rings & $10 \%$ & 5.4 & 3.1 & 0.0083 & $9.23 \mathrm{e}-12$ \\
Heartwood & $5 \%$ & 5.22 & 1.43 & 0.005 & $9.23 \mathrm{e}-12$ \\
Bark & $0 \%$ & 5 & 0 & 0 & $9.23 \mathrm{e}-12$ \\
\hline
\end{tabular}
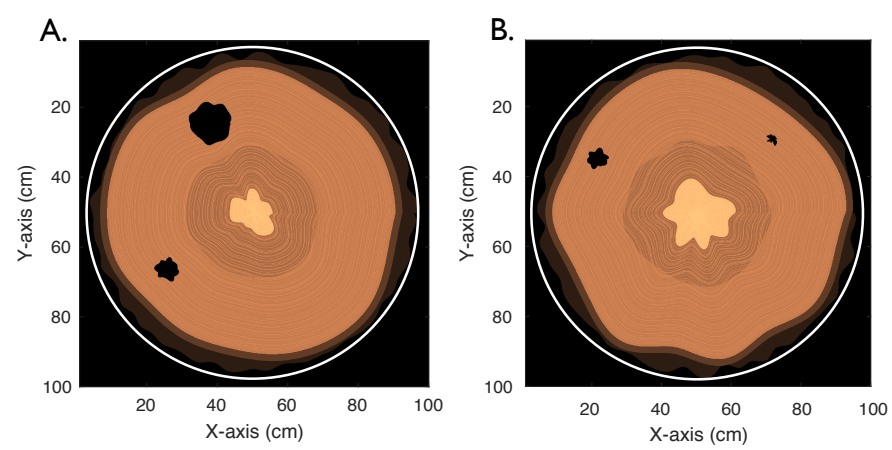

Fig. 2. The simulated trees (A, B) represent generic semi-saturated hardwoods with both inner/outer sapwood and a dry heartwood at the centre. The decay are hollow complex-shaped voids with varying sizes placed within the outer sapwood. The shape of the bark does not deviate much from a circle and therefore the measurements for the current case studies follow a circular configuration (white lines).

Consequently, the revised impressed sources are implemented as $z$-polarized soft line-sources $J_{z}(\mathbf{F}, t)=W\left(\mathbf{F}, t_{\max }-t\right)$ and the resulting migrated image equals with $Q\left(\mathbf{r}_{m}\right)=E_{z}\left(\mathbf{r}_{m}, 0\right)$.

RT-migration using FDTD is subjected to large numerical errors due to the low velocities needed to be implemented (half the estimated velocity) [60]. Numerical dispersion is proportional to the implemented permittivity [60] and can be reduced either through increasing the order of accuracy or decreasing the discretization step [71]. To that extend, for all the case studies examined in this paper, a small discretization step is chosen $\Delta x=\Delta y=1 \mathrm{~mm}$. and the time step $\Delta t$ is calculated subject to the Courant stability conditions [60]. Consequently, each A-Scan of $W\left(\mathbf{F}, t_{\max }-t\right)$ is interpolated in time in order to be synchronized with the employed $\Delta t$. To repress boundary effects, FDTD is effectively truncated using the time-synchronized convolutional perfectly matched layer (PML) [72] with ten-layer thickness.

\section{Auto-focusing}

Applying migration to dense measurements subject to an accurate estimation of the bulk permittivity should result to a sharp and focused image [58]. Therefore, there is an underlying relationship between the sharpness of the migrated image and the estimated velocity of the medium [58]. This rational is exploited in auto-focusing methods in which the migration is executed for a range of $\epsilon$ and the sharpness for each migrated image is then calculated. The permittivity that results to the most focused-sharp image is used to approximate the bulk permittivity of the host medium [58]. 
A.

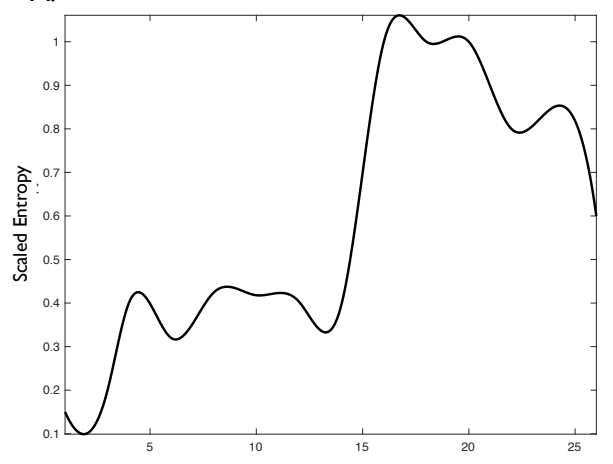

B.

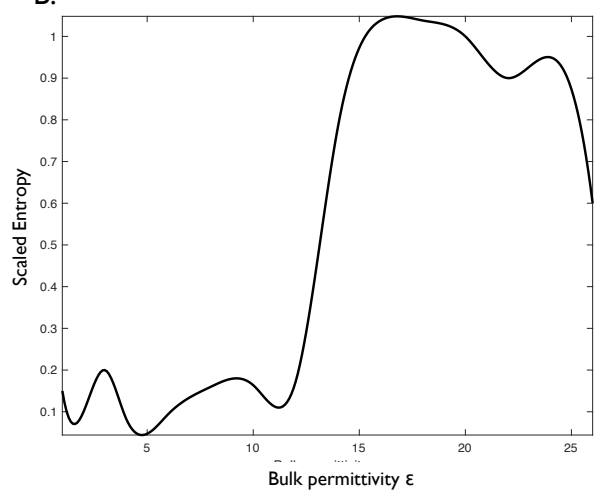

\section{A.}
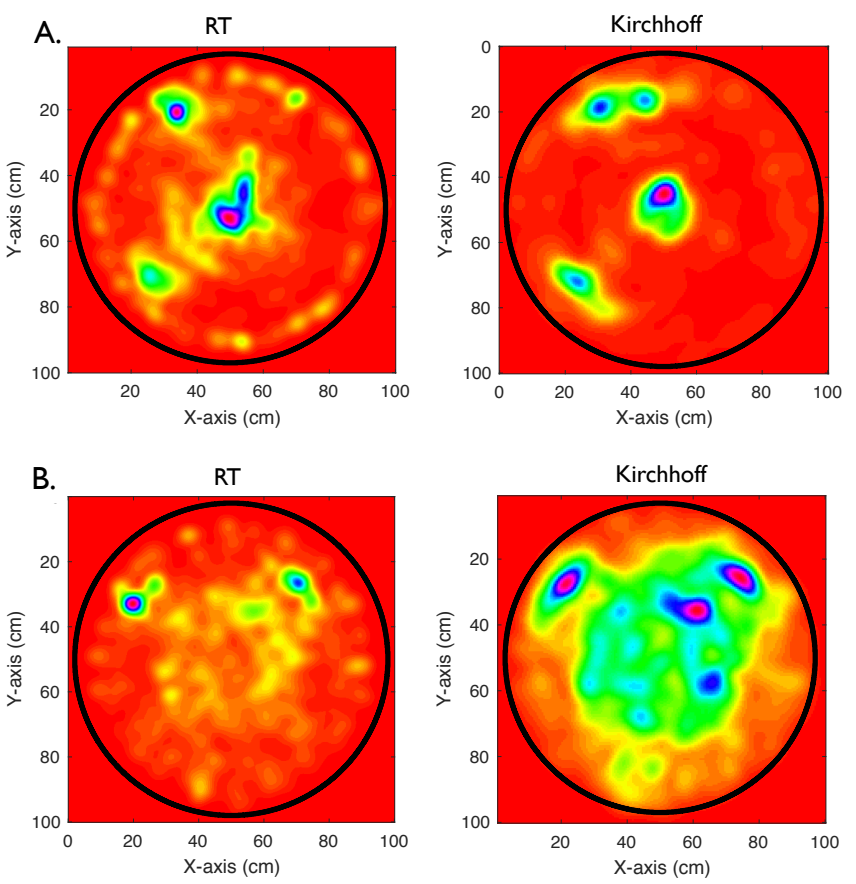

Fig. 4. The reconstructed images using both the modified Kirchhoff and the RT migration for the case studies A) and B) illustrated in Figure 2. The processing is based on circular measurements along the solid black lines.

Fig. 3. The scaled entropy for the two synthetic case studies illustrated in Figure 2. The global maximum for both of the tree samples occurs at $\epsilon \approx 17$.

Estimating the sharpness of an image is not a trivial task and different focal criteria have been reported in the literature [58]. For the case studies presented in this paper, a scaled entropy coupled with Kirchhoff migration is proven an accurate and reliable criterion for estimating the bulk permittivity of the host medium. The scaled entropy is proportional to the sharpness of the image and is calculated via

$$
F=\frac{\max Q\left(\mathbf{r}_{m}\right)}{\sum_{i=1}^{n} p_{i} \log _{2}\left(p_{i}\right)}
$$

where $\left\{p_{i} \in \mathbb{R} \mid p_{i}>0 \forall i\right\}$ is the histogram values of the investigated image $Q\left(\mathbf{r}_{m}\right)$ and $n$ is the total number of histograms $\mathbf{r}_{m}$. Prior to auto-focusing, the pre-processing step should be applied in order to remove unwanted clutter and ringing noise. The latter can give rise to artifacts that can compromise the accuracy and reliability of (9).

Figure 1 illustrates how the estimated permittivity affects $F$ in (9). Two numerical studies are examined in which three low-dielectric targets with $\epsilon=2$ are incorporated in a homogeneous medium. In the first case, the permittivity of the medium is $\epsilon=7$ and in the second case $\epsilon=14$. A 2D-FDTD is used for the simulations with $\Delta x=\Delta y=1 \mathrm{~mm}$ and $\Delta t$ follows the Courant stability condition [60]. Circular measurements are taken every four degrees using a mono-static configuration with central frequency equals to $1.5 \mathrm{GHz}$. The proposed detection scheme is applied to the raw data (pre-processing, migration, post-processing). The modified Kirchhoff migration is employed using different permittivity values varying from $\epsilon \in[1,18]$ and the scaled entropy $F$ is then calculated for each $\epsilon$. From Figure 1 it is evident that the scaled entropy is maximized near the proximity of the actual permittivity supporting the validity of the proposed focal criterion (9). The permittivity in the second example (B. in Figure 1) is slightly overestimated ( $\epsilon=15$ instead of $\epsilon=14)$ due to the numerical dispersion inherited in the FDTD-based synthetic data [60]. Numerical dispersion is more dominant in low velocity media and thus it does not affect the first example (A. in Fig. 1). From Fig. 1 it is also apparent that apart from the main peak at $\epsilon \approx 7$, there is a smaller one at $\epsilon \approx 17$. The local maximal are model-dependent fluctuations resulting from the interpretation of multiple hyperbolas under a specific velocity background as part of one hyperbola in a different velocity medium. The suggested auto-focusing criterion is based on the global maximum and model-dependent fluctuations and local maximal should be ignored.

\section{NUMERICAL EXPERIMENTS}

Trees are complex media with different shapes and sizes that often consist of five distinct layers [73], i.e. the bark, the phlem, the cabdium cell layer, the sapwood (outer and inner) and the heartwood [73], [74]. Regarding their dielectric properties, tree-layers can be seen as two-phased materials consisted of water and foliage matter [43], [75]. Consequently, both bipolar relaxation mechanisms (from water) and dispersionless components (from dry foliage) are expected to be present within the trunk [74], [76], [77]. In that context, a complex refractive index model (CRIM) [78] is suggested in [43] that estimates the complex bulk permittivity of the layers based on their water volumetric fraction. In order for the CRIM 

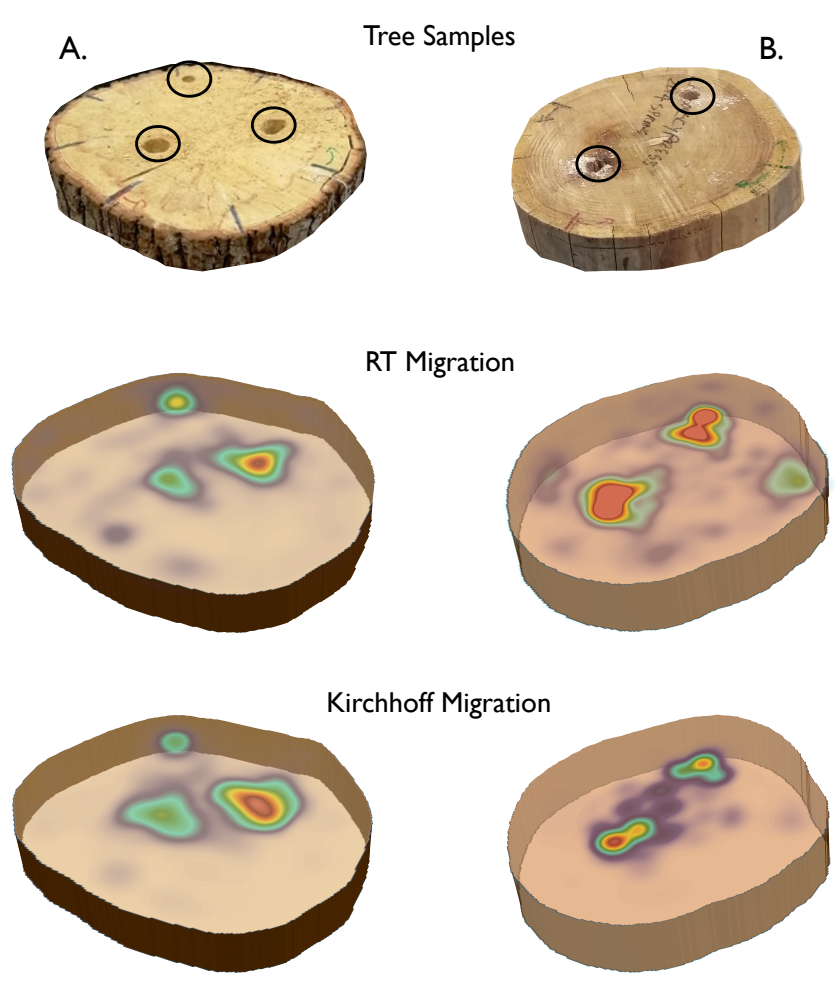

Fig. 5. The investigated tree samples and their reconstructed internal structure using the modified Kirchhoff and RT migration. The circumference of both tree samples is approximately $\approx 35-45 \mathrm{~cm}$. Circles indicate the positions of the artificially drilled decay. The decay are filled with saturated sawdust to simulate the liquid-filled chambers present in AOD-infected trees [43].

model to be compatible with FDTD, the bulk permittivity is approximated with a single Debye pole [43] using the hybrid optimization scheme proposed in [79]. Table I shows the extended Debye media that are used to fit the resulting complex permittivity for each layer. Similar to [43], the water fraction and the resistivity for each layer are chosen such as to resemblance a saturated hardwood or a semi-dry softwood [31], [80].

Two numerical case studies are examined in this section in order to assess the capabilities of the proposed approach (see 2). In both examples, two complex-shaped decay with different shapes and sizes are incorporated in the outer layers of the trunk. Circular measurements are taken every two degrees using a mono-static ideal Hertzian dipole with 1.5 $\mathrm{GHz}$ central frequency. The simulations are executed using a TM-FDTD with $\Delta x=\Delta y=1 \mathrm{~mm}$ and $\Delta t=2.357 \mathrm{ps}$ (Courant limit [60]). The Debye poles are implemented using the polarization density method [81] and the boundaries of the grid are truncated using the semi-implicit PML [72].

The first case study (see Figure 2) examines the capabilities of the proposed methodology on detecting prominent decay present on the outer sapwood. Prior to migration, a linear gain and an SVD filter are applied to the raw data (pre-processing step). The SVD filter is set to four eigenvalues. Using the proposed auto-focusing criterion (9) for the permittivity range $\epsilon=1-26$, the bulk permittivity of the trunk has been estimated at the proximity of $\epsilon \approx 17$ (see Figure 3 ). Based
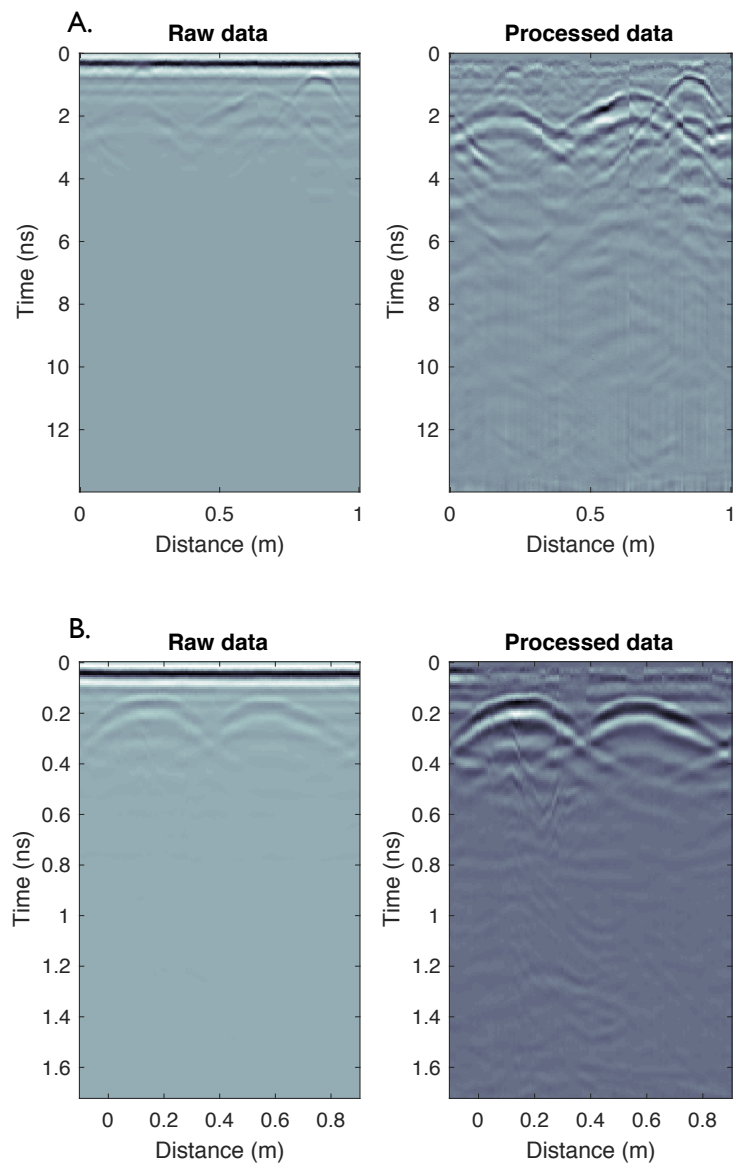

Fig. 6. The raw and processed data for the tree samples (A, B) illustrated in Figure 5. The processing pipeline consists of zero-time removal, zero-offset removal, linear gain and SVD filter removing the two dominant eigenvalues.

on that, the Kirchhoff migration has been applied assuming a homogeneous medium with $\epsilon=17$. The RT migration has the ability to incorporate any arbitrary permittivity distribution. Thus, the shape of the trunk is implemented in the model while keeping its interior homogeneous with $\epsilon=17$. Figure 4 illustrates the reconstructed internal structure of the trunk after migration and post-processing (squared and smoothed using Gaussian blur filter). It is evident that both of the decay and the heartwood are accurately detected using the modified Kirchhoff migration. RT migration detects the investigated decay and sufficiently maps the heartwood and the inner sapwood. Nonetheless, the reconstructed image using RT migration is corrupted with clutter distributed in the outer layers of the trunk. The clutter is due to the un-filtered ringing noise and cross-coupling phenomena which -as it is shown in Figure 4- do not seem to considerably affect the performance of the modified Kirchhoff migration.

The second case study (see Figure 2) investigates the ability of the current framework on detecting small decay as a manifestation of early stages of EIDs. Detecting early decay is particularly challenging due to the low signal to clutter ratio making interpretation of $Q_{\mathbf{r}_{m}}$ problematic. In order to tackle this, an SVD filter is applied prior to migration removing the twelve most dominant eigenvalues. This exhaustive SVD 


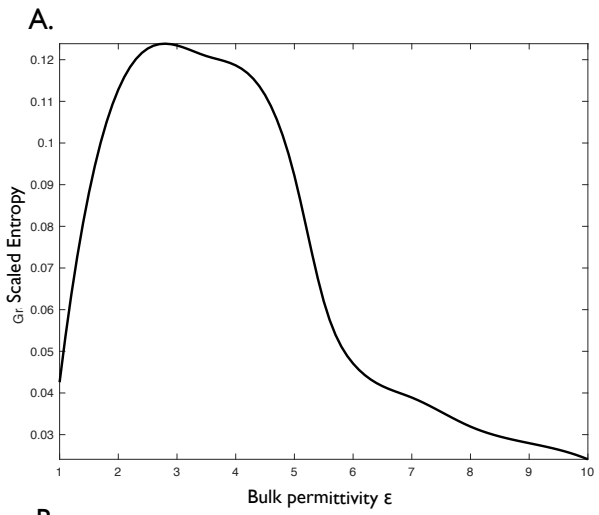

B.

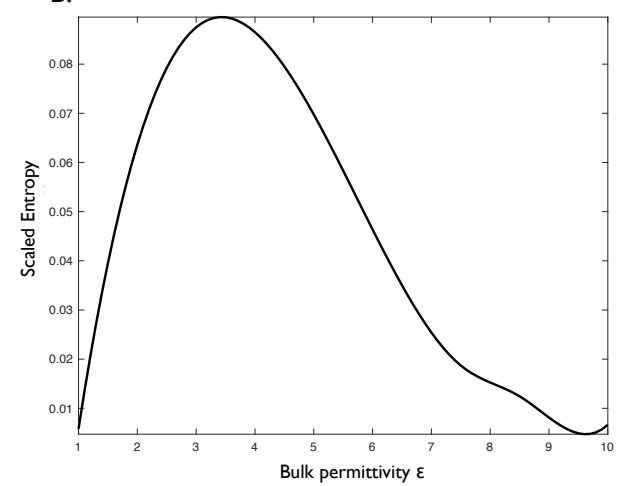

Fig. 7. The scaled entropy for the two tree samples illustrated in Figure 5. The global maximum occurs at $\epsilon \approx 3$ and $\epsilon \approx 3.5$ for the A and B sample respectively.

approach should be applied with cautious since it filtersout large targets and spatially correlated reflections resulting from targets close to the centre of the trunk. Nonetheless, it is necessary for the investigated case study in order to further reduce ringing noise, enhance the weak reflections from early decay and increase the overall signal to clutter ratio. Similar to the first example, using the proposed autofocusing criterion for the permittivity range $\epsilon=1-26$, the bulk permittivity of the tree is estimated approximately $\epsilon \approx 17$ (see Figure 3 ). Figure 4 illustrates the results using both the modified Kirchhoff and RT migration. Indications for the presence of two targets are given using both approaches. Nonetheless, it is apparent that RT outperforms the modified Kirchhoff migration providing with a more detailed and clear reconstruction of the early decay.

\section{LABORATORY MEASUREMENTS}

The proposed methodology is now tested on real measurements collected at The Lord Faringdon Centre at the University of West London (UWL). The two investigated trees are shown in Figure 5. The tree-samples have approximately the same size (diameter $\approx 35-45 \mathrm{~cm}$ ) and contain artificially created decay. The latter were filled with saturated sawdust in an effort to simulate liquid-filled chambers, a typical symptom of AOD [43]. All the artificially created decay extend parallel to the main axis of the trunks and orthogonal to the curve of acquisition $\left(T_{0}\right)$. Circular measurements were taken using the Aladdin $2 \mathrm{GHz}$ hand-held $\mathrm{CO}$ antenna from IDS
GeoRadar (Part of Hexagon). Aladdin is a commercial dualpolarized antenna that utilizes a wheel-based measuring device to position each A-Scan. Therefore, an arc-parameterization -as described in [43]- was used for positioning each AScan on the irregular $T_{0}$. The resulting scattering field from a cylindrical target is maximized when the main axis of the cylinder is aligned with the polarization of the antenna [82]. Therefore, in an effort to maximize the overall signal, the antenna polarization chosen for the investigated case studies was perpendicular to $T_{0}$ and parallel to the artificial decay. The raw data were subjected to time-zero correction, zero-offset removal, linear-gain and an SVD filter (pre-processing step). The SVD filter was set to two dominant eigenvalues. Figure 6 illustrates the raw and processed data for the investigated case studies. In order to further enhance the post-migrated signal and facilitate interpretation, the reconstructed $Q\left(\mathbf{r}_{m}\right)$ is squared and smoothed using a Gaussian blur filter (postprocessing step).

The tree sample used for the first case study is shown on the right side of Fig. 5. Two cylindrical holes were drilled with three centimeter diameter. For the second case study (left side of Figure 5), one decay with two centimeter diameter and two decay with four centimeter diameter were drilled. The tree samples were relatively dry and the bulk permittivities (estimated using the suggested auto-focussing criterion for the range $\epsilon=1-10$ ) are $\epsilon=3.5$ and $\epsilon=4$ for the first (A) and the second (B) sample respectively (see Fig. 7). Figure 5 shows the reconstructed decay using both RT and the modified Kirchhoff migration. It is apparent that the suggested detection scheme manages to adequately reconstruct the artificial decay in a clear, efficient and semi-automatic manner. Clutter is sufficiently repressed and both large and early decay are clearly detectable. Similar to the numerical experiments discussed in the previous section, the modified Kirchhoff migration is not affected by the remaining ringing noise while RT migration produces minor artifacts near the surface of the trunk.

\section{CONCLUSIONS}

Through numerical and laboratory experiments we have supported the premise that common-offset $(\mathrm{CO})$ ground penetrating radar (GPR) has the potential to become a coherent tool for early detection of tree-decay as a manifestation of emerging infectious diseases (EIDs). A novel processing scheme is described that uses a scaled Kirchhoff and a reversetime migration in order to effectively evaluate the internal structure of the tree subject to a homogeneous velocity. The latter is estimated using a modified focal criterion that calculates the bulk permittivity based on the sharpness of the migrated image. The proposed framework can be applied in a straightforward manner using any commercial GPR system with minimum computational and operational requirements in the field. This makes it particularly appealing for large-scale forestry applications and is a step forward to commercialization of GPR as a diagnostic tool against EIDs. 


\section{ACKNOWLEDGMENTS}

The authors would like to express their sincere thanks and gratitude to the following trusts, charities, organizations and individuals for their generosity in supporting this project: Lord Faringdon Charitable Trust, The Schroder Foundation, Cazenove Charitable Trust, Ernest Cook, Sir Henry Keswick, Ian Bond, P. F. Charitable Trust, Prospect Investment Management Limited, The Adrian Swire Charitable Trust, The John Swire 1989 Charitable Trust, The Sackler Trust, The Tanlaw Foundation and The Wyfold Charitable Trust.

This paper is dedicated to the memory of Jonathon West, a friend, a colleague, a forester, a conservationist and an environmentalist who died following an accident in the woodland that he loved.

\section{REFERENCES}

[1] A. Broome, D. Ray, R. Mitchell and R. Harmer, "Responding to ash dieback (Hymenoscyphus fraxineus) in the UK: woodland composition and replacement tree species," Forestry, An International Journal of Forest Research, vol. 92, pp. 108-119, 2019.

[2] A. Santini, L. Ghelardini, C. De Pace, [...] and J. Stenlid, "Biogeographical patterns and determinants of invasion by forest pathogens in Europe," New Phytologist, vol. 197, pp. 238-250, 2012.

[3] T. Jung, "Beech decline in central Europe driven by the interaction between Phytophthora infections and climatic extremes," For. Path., vol. 39, pp. 73-94, 2009

[4] A. M. Ellison, M. S. Bank, B. D. Clinton, [...] and J. R. Webster, "Loss of foundation species: consquences for the structure and dynamics of forested ecosystem," The Ecological SOciety of America, vol. 3, pp. 479486, 2005.

[5] S. A. Anagnostakis, "Chestnut blight: the classical problem of an introduced pathogen," Mycologia, vol. 79, pp. 23-37, 1987.

[6] J. Brunet, Y. Bukina, P. O. Hedwall, E. Holmstrom and G. von Oheimb, "Pathogen induced disturbance and succession in temperate forests: Evidence from a 100-year data set in souther Sweden," Basic and Applied Ecology, vol. 15, pp. 114-121, 2014.

[7] Q. Guo, M. Rejmanek and J. Wen, "Geographical, socioeconomic, and ecological determinants of exotic plant naturalization in the United States: insights and updates from improved data," NeoBiota, vol. 12, pp. 41-55, 2012.

[8] M. C. Fisher, A. D. Henk, C. J. Briggs, J. S. Brownstein, L. C. Madoff, S. H. McCraw and S. J. Gurr, "Emerging fungal threats to animal, plants and ecosystems," Nature, vol. 484, pp. 186-194, 2012.

[9] J. J. Stocks, R. J. A. Buggs and S. J. Lee, "A first assessment of Fraxinus excelsion (common ash) susceptibility to Hymenoscyphus fraxineus (ash dieback) throughout the British Isles," Nature, Scientific Reports, vol. 7, 2017.

[10] E. D. Kjaer, L. V. McKinney, L. R. Nielsen, L. N. Hansen and J. K. Hansen, "Adaptive potetial of ash (Fraxinus excelsion) populations against the novel emerging pathogen Hymenoscyphus pseudoalbidus," Evolutionaly Applications, vol. 5, pp. 219-228, 2011.

[11] S. Denman, N. Brown, S. Kirk, M. Jeger and J. Webber, "A description of the symtpoms of Acute Oak Decline in Britain and a comparative review on causes of similar disorders on oak in Europe," Forestry, vol. 87, pp. 535-551, 2014.

[12] N. Brown, D. J. G. Inward, M. Jeger and S. Denman, "A review of Agrilus biguttatus in UK forests and its relationship with acute oak decline," Forestry, vol. 0, pp. 1-11, 2014.

[13] L. Bosso, D. Russo, M. D. Febbraro, G. Cristinzio and A. Zoina, "Potential distribution of Xylella fastidiosa in Italy: a maximum entropy model," Phytopathologia Mediterranea, vol. 55, pp. 62-72, 2016.

[14] J. D. Janse and A. Obradovic, "Xylella Fastidiosa: its biology, diagnosis, control and risks," Journal of Plant Pathology, vol. 92, pp. S1.35-S1.48, 2010.

[15] E. Cella, S. Amge;etti, M. Fogolari, R. Bazzardi, L. D. Gara and M. Ciccozzi, "Two different Xylella fastidiosa strains circulating in Italy: phylogenetic and evolutoonaly analyses," Journal of Plant Interactions, vol. 13 , pp. $428-432,2018$.

[16] A. Abbott, "Italy's olive crisis intensifies as deadly tree disease spreads," Nature, vol. 563, pp. 306-307, 2018.
[17] P. J. Zarco-Tejada, C. Camino, P. S. A. Beck, R. Calderon [...] and J. A. Navas-Cortes, "Previsual symptoms of Xylella fastidiosa infeciton revealed in spectral plant-trait alterations," Nature Plants, vol. 4, pp. 432439, 2018.

[18] W. C. Shortle, K. R. Dudzik, Wood Decay in Living and Dead Trees: A Pictorial Overview, U.S. FOREST SERVICE, 2012.

[19] D. Gramaje, C. A. Brisach, A. P. Sierra, E. Moralejo, D. Olmo, L. Mostert, U. Damm and J. Armengol, "Fungal trunk pathogens associated with wood decays of almond trees on Mallorca (Spain)," Persoonia, vol. 28 , pp. 1-13, 2012.

[20] B. Larsson, B. A. Bengtsson and M. Gustafsson, Non Destructive Detection of Decay in Living Trees, Technical Report LUTEDX/(TEAT7111)/1-15/(2002); vol. TEAT-7111, 2002.

[21] A. L. Shigo, Compartmentalization of Decay in Trees, Forest Service, U. S Department of Agriculture, 1977.

[22] J. Schumacher, R. Kehr and S. Leonhard, "Mycological and histological investigations of Fraxinus excelsior nursery saplings naturally infected by Chalara fraxinea," Forest Pathology, vol. 40, pp. 419-429, 2010.

[23] L. V. McKinnery, L. R. Nielsen, D. B. Collinge, I. M. Thomsen, J. K. Hansen and E. D. Kjaer, "The ash dieback crisis: variation in resistance can prove a long-term solution," Plant Pathology, vol. 63, pp. 485-499, 2014.

[24] P. M. W. Xu and R. Wimmer, "Application of a drill resistance technique for density profile measurement in wood composite panels," Forest Prod. J., vol. 45, pp/ 90-93, 1995.

[25] W. Moore, "The combined use of the RESISTOGRAPH and the Shigometer for the accurate mapping and diagnosis of the internal condition of wood supoport orangs of trees," Arboricultural J., vol. 23, pp. 273-287, 1999.

[26] P. Niemz and D. Mannesm, "Non-destructive testing of wood and woodbased materials," J. Cult. Heritage, vol. 13, pp. S26-S34, 2012.

[27] V. Bucur. Nondestructive Characterization and Imaging of Wood. Berlin, Germany: Springer, 2003.

[28] C. J. Lin and T. H. Yang, "Detection of acoustic velocity and electrical resistance tomographies for evaluation of peripheral-inner wood demarcation in urban royal palms," Urban Forestry and Urban Greening, vol. 14 , pp. 583-589, 2015.

[29] S. A. al Hagrey, "Geophysical imaging of root-zone, trunk and moisture heterogeneity," Journal of Experimental Botany, vol. 58, pp. 839-854, 2007.

[30] D. Bieker, R. Kehr, G. Weber and S. Rust, "Non-destructive monitoring of early stages of white rot by Trametes versicolor in Fraxinus excelsior," Ann. For. Sci., vol. 67, pp.210p2- 210p7, 2009.

[31] A. Guyot, K. T. Ostergaard, M. Lenkopane, J. Fan and D. A. Lockington, "Using electrical resistivity tomography to differentiate sapwood from heartwood: applicaiton to connifers," Tree Physiology, vol. 33, pp. 187194, 2013.

[32] L. Lie and G. Li, "Acoustic tomography based on hybrid wave propagation model for tree decay detection," Computers and Electronics in Agriculture, vol. 151, pp. 276-285, 2018.

[33] L. Brancheriau, A. Ghodrati, P. Gallet, P. Thaunay and P. Lasaygues, "Application of ultrasonic tomography to characterize the mechanical state of standing trees (Picea abies)," J. Phys. Conf. Ser., vol. 353, pp. 1-13, 2012.Th

[34] J. Jezova, L. Mertens and S. Lambot, "Ground-penetrating radar for observing tree trunks and other cylindrical objects," Construction and Building Materials, vol. 123, pp. 214-225, 2016.

[35] J. Jeov, S. Lambot, A. Fedeli and A. Randazzo, "Ground-penetrating radar for tree trunk investigation," in Proc of 9th International Workshop on Advanced Ground Penetrating Radar (IWAGPR), Edinburgh, pp. 1-6, 2017.

[36] J. R. Butnor, M. L. Pruyn, D. C. Shaw, M. E. Harmon, A. N. Mucciardi and M. G. Ryn, "Detecting defects in conifers with ground penetrating radar: applications and challenges," Forest Pathology, vol. 39, pp. 309322, 2009.

[37] J. Jezova and S. Lambot, "Influence of bark surface roughness on tree trunk radar inspection," Ground Penetrating Radar, vol. 1, pp. 1-25, 2018

[38] J. Jezova, J. Harou and S. Lambot, "Reflection waveforms occurring in bistatic radar testing of columns and tree trunks," Construction and Building Materials, vol. 174, pp. 388-400, 2018.

[39] X. Xiao, J. Wen, Z. Xiao and W. Li, "Detecting and measuring internal anomalies in tree trunks using radar data for layer identification," Journal of Sensors, vol 2018, pp. 1-11, 2018.

[40] F. Boero, A. Fedeli, M. Lanini, M. Maffongelli, R. Monleone, M. Pastorino, A. Randazoo, A. Slvade and A. Sansalone, "Microwave Tomography for the Inspection of Wood Materials: Imaging System and 
Experimental Results," IEEE Transactions on Microwave Theory and Techniques, vol. 66, no. 7, pp. 3497-3510, July 2018

[41] L. Fu, S. Liu and L. Liu, "Internal structure characterization of living tree trunk cross-section using GPR: Numerical examples and field data analysis," in Proc. of the 15th International Conference on Ground Penetrating Radar, Brussels, 2014, pp. 155-160.

[42] D. J. Daniels, Ground Penetrating Radar, 2nd ed. London, U.K.: Institution of Engineering and Technology, 2004.

[43] I. Giannakis, G. Tosti, L. Lantini and A. Alani, "Health monitoring of tree-trunks using ground penetrating radar", IEEE Transactions on Geoscience and Remote Sensing, Early access available.

[44] O. Yilmaz, Seismic data analysis, Society of Exploration Geophysics, 2001.

[45] M. A. Gonzlez-Huici, I. Catapano and F. Soldovieri, "A Comparative Study of GPR Reconstruction Approaches for Landmine Detection," IEEE Journal of Selected Topics in Applied Earth Observations and Remote Sensing, vol. 7, no. 12, pp. 4869-4878, Dec. 2014.

[46] J. Schofield, D. Daniels and P. Hammerton, "A Multiple Migration and Stacking Algorithm Designed for Land Mine Detection," IEEE Transactions on Geoscience and Remote Sensing, vol. 52, no. 11, pp. 6983-6988, Nov. 2014

[47] X. Feng, M. Sato and C. Liu, "Hand-Held GPR Imaging Using Migration for Irregular Data," IEEE Journal of Selected Topics in Applied Earth Observations and Remote Sensing, vol. 4, no. 4, pp. 799-803, Dec. 2011.

[48] L. Lei et al., "Reverse Time Migration of Crosswell GPR Data Based on Wavefield Decomposition," in Proc. of the 17th International Conference on Ground Penetrating Radar (GPR), Rapperswil, pp. 1-4, 2018.

[49] X. Feng, M. Sato, C. Liu and Y. Zhang, "Profiling the Rough Surface by Migration," IEEE Geoscience and Remote Sensing Letters, vol. 6, no. 2, pp. 258-262, April 2009.

[50] H. Jung, W. Kang and K. Kim, "Multilayer Stolt Migration Algorithm for Subsurface Target Imaging in Oblique Layers," IEEE Journal of Selected Topics in Applied Earth Observations and Remote Sensing, vol. 10, no. 10, pp. 4295-4303, Oct. 2017.

[51] N. Bonomo, D. Bullo, A. Villela and A. Osella, "Ground-penetrating radar investigation of the cylindrical pedestal of a monument," Journal of Applied Geophysics, vol. 113, pp. 1-13, 2015.

[52] S. J. Radzevicius, B. T. Clark, D. Herbst and T. T. Webster, 'Imaging columns with GPR," in Proc. of the Tenth International Conference on Ground Penetrating Radar, Delft, Netherlands, pp. 387-390, 2004.

[53] R. Persico, G. Gennarelli and F. Soldovieri, "GPR prospecting on circular surfaces: preliminary results," in Proc. 15th International COnference on Ground Penetrating Radar Brussels, 2014.

[54] F. Lehmann and A. G. Green, "Topographic migration of georadar data: Implications for acquicition and processing," Geophysics, vol. 65, pp. 836-848, 200.

[55] J. R. Dujardin and M. Bano, "Topographic migration of GPR data Case studies from dry sand dunes and active fault areas," in Proc. of 18th European Meeting of Environmental and Engineering Geophysics, Paris, 2012.

[56] I. G. Cumming and Frank H. Wong, Digital Processing of SyntheticAperture Radar Data, Artech House Nordwood, MA, 2005.

[57] M. A. Gonzalez-Huici, "Adaptative Stolt migration via contrast maximization for GPR applications," in Proc. of the 6th International Workshop on Advanced Ground Penetrating Radar (IWAGPR), Aachen, 2011, pp. 1-5.

[58] X. Wei and Y. Zhang, "Autofocusing Techniques for GPR Data from RC Bridge Decks," IEEE Journal of Selected Topics in Applied Earth Observations and Remote Sensing, vol. 7, no. 12, pp. 4860-4868, Dec. 2014.

[59] K. S. Yee, "Numerical Solution of Initial Boundary Value Problems Involving Maxwell's Equations," IEEE Trans. on AP, vol. 14, no. 3, pp. 302-307, May 1966

[60] A. Taflove and S. C. Hagness, Computational Electrodynamics, the Finite-Difference Time-Domain Method, 2nd ed. Norwood, MA, USA: Artech House, 2000

[61] C. J. Leuschen and R. G. Plumb, "A matched-filter-based reverse-time migration algorithm for ground-penetrating radar data," IEEE Transactions on Geoscience and Remote Sensing, vol. 39, no. 5, pp. 929-936, May 2001.

[62] E. Fischer, G. A. McMechan, A. P. Annan and S. W. Cosway, 'Examples of reverse-time migration of single-channel, ground-penetrating radar profiles," Geophysics, vol. 57, pp. 577-586, 1992.

[63] H. Liu, Z. Long, F. Han, G. Fang and Q. H. Liu, "Frequency-Domain Reverse-Time Migration of Ground Penetrating Radar Based on Layered Medium Green's Functions," IEEE Journal of Selected Topics in Applied
Earth Observations and Remote Sensing, vol. 11, no. 8, pp. 2957-2965, Aug. 2018.

[64] H. Kim, S. J. Cho, and M. J. Yi, "Removal of ringing noise in GPR data by signal processing," Geosci. J. , vol. 11, pp. 75-81, Mar. 2007.

[65] M. S. Nixon and A. S. Aguado, Feature Extraction and Image Processing. Academic Press, 2008, p. 88.

[66] R. J. Sharpe, R. W. Thorpe, "Numerical Method for Extracting an Arc Length Parameterization from Parametric Curves", Computer Aided Design, vol. 12, no. 2, pp. 79-81, March 1982.

[67] B., Guenter and R. Parent, "Computing the arc length of parametric curves,-IEEE Comp. Graph. Appl. , vol. 10, no. 3, pp. 72-78, 1990.

[68] I. Giannakis, S. Xu, P. Aubry, A. Yarovoy and J. Sala, "Signal processing for landmine detection using ground penetrating radar," in Proc. of the IEEE International Geoscience and Remote Sensing Symposium (IGARSS), Beijing, 2016, pp. 7442-7445.

[69] E. Kreyszig, Advanced Engineering Mathematics, 8th ed. New York, NY, USA: Wiley, 1999.

[70] C. Balanis, Advanced Engineering Electromagnetics, Wiley New York, 1989.

[71] V. Kantartzis and T. D. Tsiboukis, Higher Order FDTD Schemes for Waveguide and Antenna Structures, 1st edition, Morgan and Claypool publishers.

[72] I. Giannakis and A. Giannopoulos, "Time-Synchronized convolutional perfectly matched layer for improved performance in FDTD," IEEE Antennas and Wireless Propagation Letters, vol. 14, pp. 690-693, 2015.

[73] T. Russel and C. Cutler, Trees, an illustrated identifier and encyclopedia, Hermes House, Leicestershire, 2012

[74] T. Douglas, Effective Dielectric Constants of Foliage Media, Rome Air Development Center Air Force Systems Command Grifflss Air Force Base, Rome, 1990.

[75] M. G. Broadhurst, "Complex Dielectric Constants and Dissipation Factor of Foliage," NBS Report no. 9592, NBS project 3110107, US Naval Ordnance Laboratory, October 1970.

[76] G. S. Brown, and W. J. Curry, "A Theory and Model for Wave Propagation Through Foliage", Radio Science, vol. 17, pp. 1027-1036, 1982.

[77] W. L. James, "Dielectric Properties of Wood and Hardboard: Varaition With Temperature, Frequency, Moisture Content and Grain Orientation," USDA Forest Service Res. Paper FPL 245, USDA Forest Products Lab. Madison, MI, 1975.

[78] R. J. Birchak, C. G. Gardner, E. J. Hipp, and M. J. Victor, "High dielectric constant microwave probes for sensing soil moisture," in Proc. of the IEEE, vl. 62, no. 1, pp 93-98, 1974.

[79] D. F. Kelley, T. J. Destan, and R. J. Luebbers, "Debye function expansions of complex permittivity using a hybrid particle swarm-leas squares optimisation approach," IEEE Trans. Antennas Propag. , vol. 55, no. 7, pp. 1999-2005, Jul. 2007

[80] N. Brazee, R. Marra, L. Goecke and P. Van Wassenaer, "Non-destructive assessment of internal decay in three hardwood species of northeastern North America using sonic and electrical impedance tomography," Forestry, vol. 84, pp. 33-39.

[81] I. Giannakis and A. Giannopoulos, "A novel piecewise linear recursive convolution approach for dispersive media using the finite-difference time-domain method," IEEE Trans. Antennas Propag., vol. 62, no. 5, pp. 2669-2678, May 2014

[82] S. J. Radzevivious and J. J. Daniels, "Ground penetrating radar polarisation and scattering from cylinders," Journal of Applied Geophysics, vol. 45 , pp. 111-125, 2000/ 


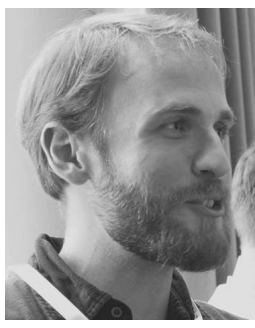

Iraklis Giannakis received the bachelors and masters degrees in geophysics from the Aristotle University of Thessaloniki, Thessaloniki, Greece, in 2009 and 2011, respectively, and the Ph.D. degree from The University of Edinburgh, Edinburgh, U.K., through a Project co-funded by the Defence Science and Technology Laboratory, U.K., and the Engineering and Physical Sciences Research Council, U.K. $\mathrm{He}$ is currently a Research Fellow with the School of Computing and Engineering, University of West London, London, U.K., where he has been involved in applications of ground-penetrating radar (GPR) to sustainable management of forestry heritage. His research interests include near-surface geophysics, nondestructive testing, computational e lectromagnetics, and machine learning. Dr. Giannakis received the Best Paper Award at the 15th International Conference on GPR in 2014, during his Ph.D. degree.

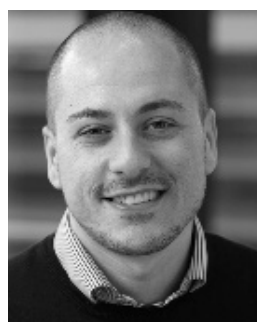

Fabio Tosti received his MSc Engineering degree cum laude in Infrastructure and Transportation Engineering at Roma Tre University, Italy, in 2010, where he also he received his PhD with Doctorate (excellent rating), in 2014. He is currently an Associate Professor in Civil Engineering and Deputy Head of The Faringdon Centre The Non-destructive Testing Centre.

Dr Tosti's research interests include the development of new algorithms, methodologies and numerical models for geoscience applications and the nondestructive assessment, repair and maintenance of civil engineering infrastructures. He has over 120 research publication records in international journals, conferences and books and has served as Associate Editor and Managing Guest Editor in numerous international journals.

He served as Organizer, Panel Member (Scientific), and Session Chair in several international conferences and held a supervisory role at the international project European Cooperation in Science and Technology TU1208 Action, titled "Civil Engineering Applications of Ground Penetrating Radar".

In 2017 Dr Tosti was granted an Early Career Scientists Award by the European Geosciences Union (EGU) for his outstanding contribution to the development of new GPR methodologies in geosciences and civil engineering. Additionally he holds the post of Convener at the EGU General Assembly for sessions on NDT methods and data fusion.

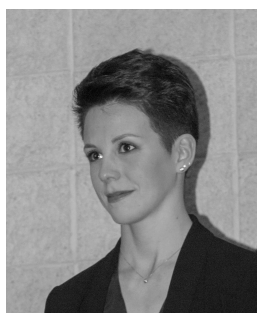

Livia Lantini received her BSc (Hons) degree in Civil Engineering and her MSc degree in Infrastructure and Transportation Engineering at the Department of Engineering Roma Tre University, Italy. Since 2018 she is a PhD student at the School of Computing and Engineering of the University of West London. Her research focuses on the assessment and health monitoring of trees and the investigation of tree roots and soil interaction using Ground Penetrating Radar and other non-destructive methods.

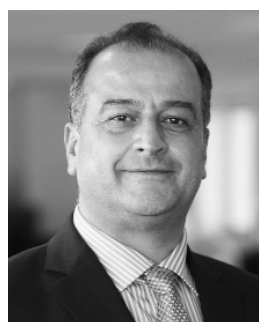

Amir Alani joined the University of West London, UK, in 2014. In addition to his position as the Executive Dean of the School of Computing and Engineering, he leads the Faringdon Centre (the Non-Destructive Research Centre) at the University.

Professor Alani is an expert in the assessment, repair, and maintenance of civil engineering infrastructures such as bridges, tunnels, and buildings and has extensive work experience with different professional bodies in the fields of engineering and design both in the UK and Europe. He was appointed as the Rochester Bridge Trust Professor of Engineering in 2018 and has more than 25 years work experience in Higher Education and industry in the UK.

With an established track record of research and development in different branches of civil and mechanical engineering, including the applications of non-destructive testing methods, concrete technology, geotechnics and soil engineering and engineering education, Professor Alani has published over 180 journal papers, book chapters, international conference papers, and consulting external reports in these areas.

Professor Alani was a Management Committee Member for a European Cooperation in Science and Technology (COST) Action project, TU1208 Project 2012-2017, Civil Engineering Applications of Ground Penetrating Radar (GPR). He has delivered and led numerous seminars and workshops nationally and internationally, and has chaired a number of international conference sessions in the last 20 years. 